\title{
Entrance channel effects in superheavy element production
}

\author{
Avazbek Nasirov ${ }^{1,2, a}$, Giorgio Giardina ${ }^{3,4,5, \mathrm{~b}}$, Giuseppe Mandaglio ${ }^{3,4,5, \mathrm{c}}$, \\ and Akhtam Muminov ${ }^{2}$ \\ ${ }^{1}$ Joint Institute for Nuclear Research, 141980 Dubna, Russia \\ ${ }^{2}$ Institute of Nuclear Physics, 100214 Tashkent, Uzbekistan \\ ${ }^{3}$ Dipartimento di Scienze Matematiche e Informatiche, Scienze Fisiche e Scienze della Terra, \\ University of Messina, 98166 Messina, Italy \\ ${ }^{4}$ INFN Gruppo Collegato di Messina, Messina, Italy \\ ${ }^{5}$ Dipartimento di Scienze Chimiche, Biologiche, Farmaceutiche ed Ambientali, University of \\ Messina, 98166 Messina, Italy
}

\begin{abstract}
The difference between evaporation residue cross sections measured in the cold $\left(\mathrm{X}+{ }^{208} \mathrm{~Pb},{ }^{209} \mathrm{Bi}\right)$ and hot $\left({ }^{48} \mathrm{Ca}+\mathrm{actinides}\right)$ fusion reactions can be related to the stage of compound nucleus $(\mathrm{CN})$ formation and/or to the stage of its survival against fission. The cold fusion reactions are favorable in synthesis of the superheavy elements (SHE) with charge numbers $Z<112$ in comparison with the hot fusion reactions due to small excitation energy and large fission barrier of the $\mathrm{CN}$ formed in these reactions. The strong decrease of the cross sections of the synthesis of the SHE $Z=113$ in the cold fusion reactions in comparison with the ones in the hot fusion reactions is the result of the increase of hindrance to the $\mathrm{CN}$ formation in the cold fusion reactions. The origin of the intrinsic fusion barrier, $B_{\text {fus }}^{*}$, causing the strong decrease of the probability $P_{\mathrm{CN}}$ in the cold fusion is discussed.
\end{abstract}

\section{Introduction}

The behaviour of the experimental cross sections obtained in synthesis of the SHE with charge numbers $Z=110-118$ can be explained by the strong role of the entrance channel characteristics and fission barrier of the $\mathrm{CN}$ in the reaction mechanism. The SHE with $Z=110-112$ were synthesized in cold fusion reactions with $\mathrm{Pb}$ and $\mathrm{Bi}$ targets which have large binding energies [1]. But the smallest cross section of the synthesis of the SHE with $Z=113$ observed in the ${ }^{70} \mathrm{Zn}+{ }^{209} \mathrm{Bi}$ [2] reaction demonstrated the hopelessness of using the cold fusion reactions to synthesize heavier $Z>113$ elements. The reactions with the projectile ${ }^{48} \mathrm{Ca}$ and actinide targets are favourable in the synthesis of the new SHE Fl, 115, Lv, 117, and $118[3,4]$.

The evaporation residue (ER) cross sections obtained at the synthesis of the SHE $Z=106-111$ in the cold fusion reactions were sufficiently larger than with the ones observed in the hot fusion reactions (see Fig. 1) since the $\mathrm{CN}$ formed in the last reaction has a large

\footnotetext{
a e-mail: nasirov@jinr.ru

b e-mail: ggiardina@unime.it

c e-mail: gmandaglio@unime.it
} 


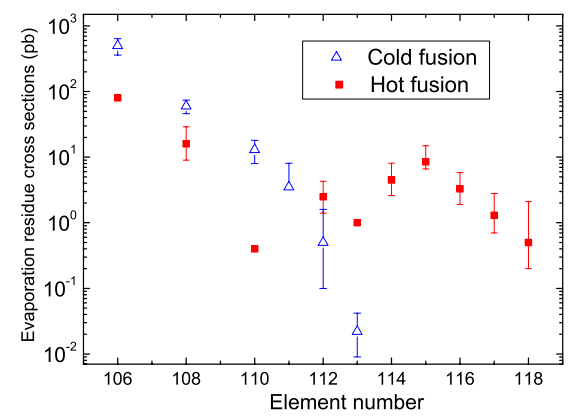

Figure 1. The comparison of the ER cross sections obtained in the cold (open triangles) and hot (solid squares) fusion reactions used to synthesize SHE.

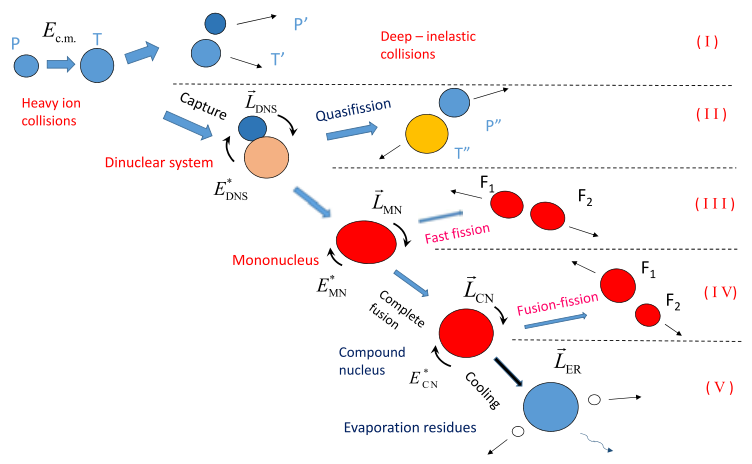

Figure 2. Sketch of reaction channels (steps I to V) leading to the formation of binary products and reducing the probability for the synthesis of superheavy elements $(\mathrm{V})$ in a collision of a projectile ion $\mathrm{P}$ with a target nucleus $\mathrm{T}$. $\mathrm{P}^{\prime}$ and $\mathrm{T}^{\prime}$ are deep-inelastic collision (incomplete momentum transfer) products, $\left(\mathrm{P}^{\prime \prime}\right.$ and $\left.\mathrm{T}^{\prime \prime}\right)$ quasifission (total momentum transfer) products and $\left(\mathrm{F}_{1}\right.$ and $\left.\mathrm{F}_{2}\right)$ fast fission and fusion-fission products.

excitation energy. Consequently, the survival probability of the very excited $\mathrm{CN}$ during deexcitation cascade is small and this fact leads to decreasing the cross section of the synthesis in the hot fusion reactions. But starting the synthesis of $\mathrm{Cn}(Z=112)$ the evaporation residues' yield in the experiments by the cold fusion became lower in comparison with the ones observed in the reactions with the ${ }^{48} \mathrm{Ca}$ projectile. This circumstance can be explained by the increase of the hindrance in formation of the $\mathrm{CN}$ in the cold fusion reactions caused by the dominance of the quasifission process.

In spite of the successes in the SHE synthesis there is no unambiguous mechanism of the complete fusion of nuclei in the heavy-ion collisions. We will discuss the processes preceding the formation of SHE, and the influence of the entrance channel on the probability of the CN formation.

A theoretical model to calculate the cross section of processes in heavy-ion collisions has been developed on the basis of the realistic concept about reaction mechanism $[5,6]$. The sequence of the stages of the heavy-ion collisions leading to the synthesis of $\operatorname{SHE}\left(\sigma_{\mathrm{ER}}\right)$ shown in Fig. 2 is calculated by the corresponding equations:

$$
\sigma_{\mathrm{ER}}(E)=\sum_{\ell=0}^{\ell_{d}(E)}(2 \ell+1) \sigma_{\mathrm{cap}}(E, \ell) P_{\mathrm{CN}}(E, \ell) W_{\text {sur }}(E, \ell),
$$


where $\sigma_{\text {cap }}, P_{\mathrm{CN}}$, and $W_{\text {sur }}$ are the capture cross section, fusion probability, and survival probability, respectively. The methods of calculation used to estimate the ER cross sections are listed here:

1. The partial capture cross sections are found by the solution of the equations of the relative and tangential motions. As a result the values of the orbital angular momentum $\left(0<\ell<\ell_{d}\right)$ contributing to the interaction of nuclei are determined [5, 7].

2. Changes of the initial charge and mass numbers of the interacting nuclei at the capture stage is calculated by the set of transport equations-master equations. The transition coefficients depend on the shell effects in nuclei due to the used microscopic method of their calculation. The method of calculation is presented in the appendix of Ref. [8].

3. The probability $P_{\mathrm{CN}}$ of the $\mathrm{CN}$ formation from the dinuclear system (DNS) state is calculated statistically by the use of the branching ratio of level densities on the Businaro-Gallone, on the minimum of the potential well and on the maximum of the way to symmetric configuration [9].

4. The ER yield after emission neutrons, $\gamma$ quanta and charged particles (protons and $\alpha$ particles) from the heated and rotating compound nucleus during de-excitation cascade is determined by the advanced statistical model $[10,11]$.

\section{The advantage of cold fusion reactions in synthesis of SHE $Z<112$}

The $\mathrm{CN}$ is formed by the transformation of the DNS in competition with the quasifission. The last process is the decay of the DNS formed at capture of the projectile nucleus by the target nucleus without reaching the CN equilibrium shape. See stage (II) in Fig. 2. The time needed to transform the DNS into a $\mathrm{CN}$ is large in the case of collision of the massive nuclei since there is the intrinsic fusion barrier which must be overcome, and the alternative quasifission process has the huge cross section and its angular distribution may be very wide $[12,13]$. But the realistic lifetime of the DNS may be not enough for the complete fusion. As a result the quasifission is the dominant process in the stage (II) of the DNS evolution to the complete fusion.

The branching ratio of the competition between complete fusion and quasifission processes is determined by the peculiarities of the DNS which is calculated as a sum of the total interaction potential $V$ of nuclei and reaction energy balance $\left(Q_{g g}\right)$ :

$$
U\left(Z_{1}, A_{1}, Z_{2}, A_{2}, \ell ; R\right)=Q_{g g}+V\left(Z_{1}, A_{1}, Z_{2}, A_{2}, \ell ; R\right)-V_{\text {rot }}^{C N}(\ell),
$$

where $A_{i}$ and $Z_{i}(i=1,2)$ are mass and charge numbers of the DNS fragments; $R$ is the internuclear distance between their centres-of-mass; $Q_{\mathrm{gg}}=B_{1}+B_{2}-B_{\mathrm{CN}}$ is the reaction energy balance; $B_{1}, B_{2}$ and $B_{\mathrm{CN}}$ are the binding energies of the interacting nuclei and $\mathrm{CN}$, respectively, which are obtained from the nuclear mass tables in references $[14,15] ; V_{\text {rot }}^{C N}(\ell)$ is the $\mathrm{CN}$ rotational energy.

The nucleus-nucleus potential $V(A, Z, \ell ; R)$ consists of the three parts:

$$
\begin{aligned}
V\left(Z_{1}, A_{1}, Z_{2}, A_{2}, \ell ; R\right)= & V_{\text {Coul }}\left(Z_{1}, A_{1}, Z_{2}, A_{2} ; R\right)+V_{\text {nucl }}\left(Z_{1}, A_{1}, Z_{2}, A_{2} ; R\right) \\
& +V_{\text {rot }}\left(Z_{1}, A_{1}, Z_{2}, A_{2}, \ell ; R\right),
\end{aligned}
$$

where $V_{\text {Coul }}, V_{\text {nucl }}$, and $V_{\text {rot }}$ are the Coulomb, nuclear, and rotational potentials, respectively. The methods of calculation of these three parts of the nucleus-nucleus potential are presented in appendix A of Refs. [5, 7]. 


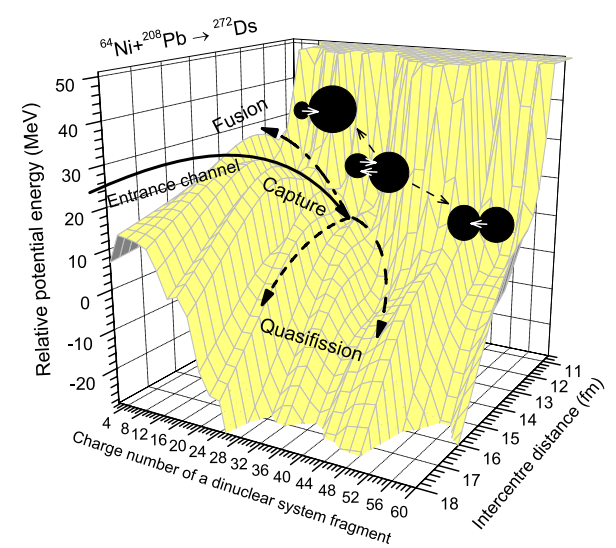

Figure 3. PES calculated for the DNS formed via ${ }^{64} \mathrm{Ni}+{ }^{208} \mathrm{~Pb}$. The arrows show conditionally directions of the DNS evolution to complete fusion and quasifission after capture in the entrance channel.

The difference in the evolution path of the DNS formed in the ${ }^{64} \mathrm{Ni}+{ }^{208} \mathrm{~Pb}$ and ${ }^{34} \mathrm{~S}+{ }^{244} \mathrm{Pu}$ reactions in the direction of the complete fusion is seen from the comparison of Figs. 3 and 4, respectively, which are presented as a function of the charge number of the DNS fragments and internuclear distance $R$ between their centres-of-masses. The position of the DNS energy path on the potential energy surface (PES) at capture (the end of the solid arrow in Figs. 3 and 4) is important for the transformation of the DNS into the CN. The capture on the PES for the ${ }^{34} \mathrm{~S}+{ }^{244} \mathrm{Pu}$ reaction $\left(Z_{p}=16\right)$ is close to the maximum (Businaro-Gallone point) on the way to complete fusion while the initial position of the ${ }^{64} \mathrm{Ni}+{ }^{208} \mathrm{~Pb}$ system at capture is placed sufficiently lower $\left(Z_{p}=28\right)$. The difference in the potential energy axis between the minimum of the potential well where the path of the relative kinetic energy trapped ( $Z_{\text {capture }} \sim Z_{\text {projectile }}$ ) and Businaro-Gallone point is used as the intrinsic fusion barrier for the complete fusion $B_{\text {fus }}^{*}$ (see Fig. 5). The appearance of the $B_{\text {fus }}^{*}$ is related to the large binding energy of nucleons in the nuclei with magic proton and neutron numbers. For the heavier and mass symmetric DNS system the depth of this valley is much lower and the value of $B_{\text {fus }}^{*}$ increases causing the hindrance to transformation of the DNS into CN through nucleon transfer.

Therefore, the fusion cross section of the ${ }^{64} \mathrm{Ni}+{ }^{208} \mathrm{~Pb}$ reaction is smaller than the one for the ${ }^{34} \mathrm{~S}+{ }^{244} \mathrm{Pu}$ reaction but the excitation energy $E_{\mathrm{CN}}^{*}$ of the $\mathrm{CN}$ formed in the latter reaction is large $\left(E_{\mathrm{CN}}^{*}>35 \mathrm{MeV}\right)$. The large excitation energy and small fission barrier for the isotopes of Ds with the neutron numbers $N=164-168$ (see Fig. 6) make the survival probability $W_{\text {sur }}(E, \ell)$ of the $\mathrm{CN}{ }^{278} \mathrm{Ds}$ formed in the ${ }^{34} \mathrm{~S}+{ }^{244} \mathrm{Pu}$ reaction very small. Consequently, the cross section of the synthesis of Ds in the latter reaction is smaller than the one obtained in the cold fusion reaction, ${ }^{64} \mathrm{Ni}+{ }^{208} \mathrm{~Pb}$ (see Fig. 1). The values of the fission barrier calculated for the isotopes of Ds and Cn by Kowal et al. [16] are presented in Fig. 6 as a function of the neutron number.

The large fission barrier and small excitation energy of the $\mathrm{CN}^{272} \mathrm{Ds}(N=162)$ formed in the ${ }^{64} \mathrm{Ni}+{ }^{208} \mathrm{~Pb}$ reaction increase very strongly its survival probability. The gain in the surviving stage $(\mathrm{V})$ of the cold fusion reaction can guarantee large cross section of the synthesis in spite of the strong hindrance to complete fusion. Figure 7 obtained from Ref. [17] demonstrates that the fusion excitation function (dashed curve) is much lower than the quasifission (dotted) curve due to the large value $B_{\text {fus }}^{*}$ in the ${ }^{64} \mathrm{Ni}+{ }^{208} \mathrm{~Pb}$ reaction. The experimental data on the synthesis of $\mathrm{Ds}$ in the ${ }^{64} \mathrm{Ni}+{ }^{208} \mathrm{~Pb}$ reaction were reproduced 


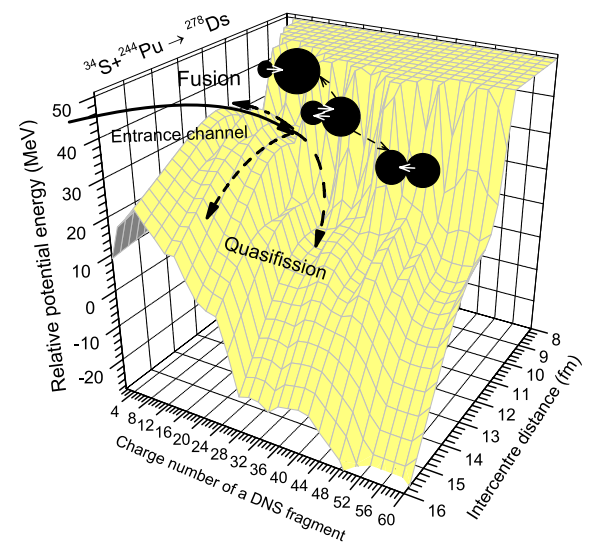

Figure 4. The same as in Fig. 3, but for the reaction ${ }^{34} \mathrm{~S}+{ }^{244} \mathrm{Pu}$.

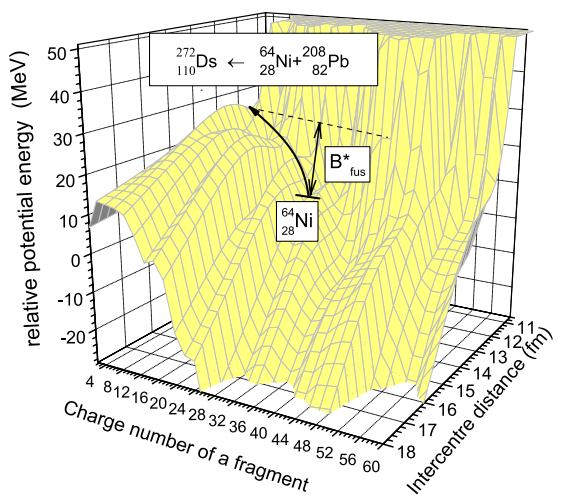

Figure 5. The intrinsic fusion barrier, $B_{\text {fus }}^{*}$, is determined by the difference between the values of the PES corresponding to the entrance channel $(Z=28)$ and its maximum value (similar to the BusinaroGallone point) in the way to fusion.

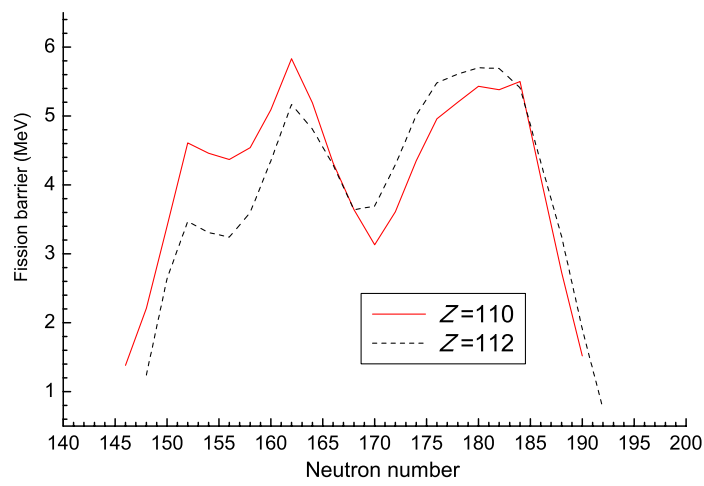

Figure 6. Fission barriers for the isotopes Ds and Cn obtained from Ref. [16] as a function of neutron numbers. 


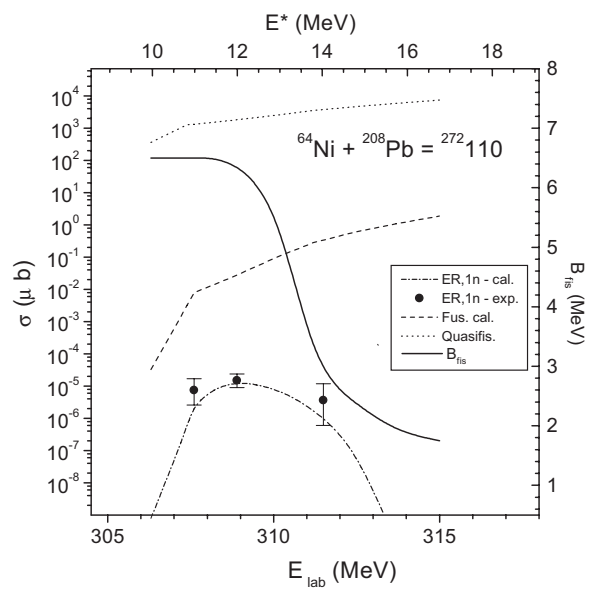

Figure 7. Theoretical quasifission (dotted curve), fusion (dashed curve) and $1 n$-evaporation residue (dot-dashed) excitation functions (left axis) for the reaction ${ }^{64} \mathrm{Ni}+{ }^{208} \mathrm{~Pb}$ obtained from Ref. [17]. The solid curve shows the fission barrier for the compound nucleus ${ }^{272} \mathrm{Ds}$ (right ordinate axis). The experimental cross sections of the evaporation residue formation in the $1 n$-channel are shown by the solid circles [1].

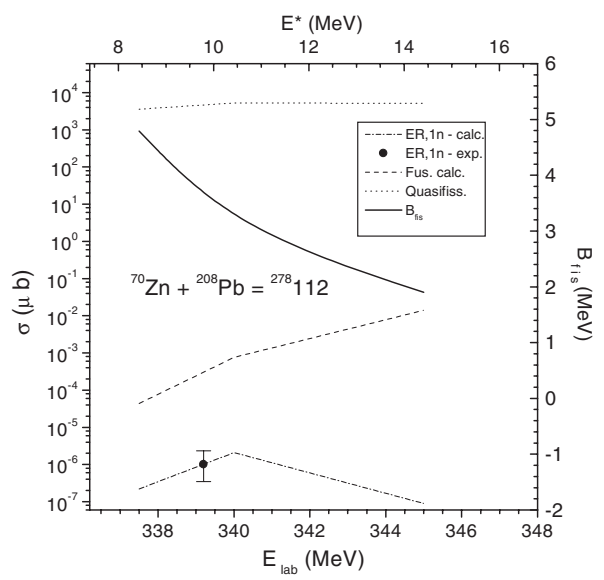

Figure 8. Same as in Fig. 7 but for the ${ }^{64} \mathrm{Ni}+{ }^{208} \mathrm{~Pb}$ reaction.

well. Nevertheless the ER cross section in this cold fusion reaction is larger than the one obtained in the hot fusion ${ }^{34} \mathrm{~S}+{ }^{244} \mathrm{Pu}$ reaction. It is seen that, the smallness of the ER cross section in the ${ }^{34} \mathrm{~S}+{ }^{244} \mathrm{Pu}$ reaction is related to the small fission barrier $(3.8 \mathrm{MeV})$ and large excitation energy of the $\mathrm{CN}$, which is formed. The restriction to the minimum value of $E_{\mathrm{CN}}$ is determined by the landscape of potential energy surface (PES) which is high in the entrance channel for the mass asymmetric ${ }^{34} \mathrm{~S}+{ }^{244} \mathrm{Pu}$ reaction.

\section{The advantage of the hot fusion reactions in synthesis of SHE $Z \geq 112$}

The hot fusion reactions with the ${ }^{48} \mathrm{Ca}$ beam are favourable in the synthesis of SHE $Z \geq 112$ (see Fig. 9). The doubly-magic ${ }^{48} \mathrm{Ca}$ is a neutron-rich isotope and its use as a beam increases 


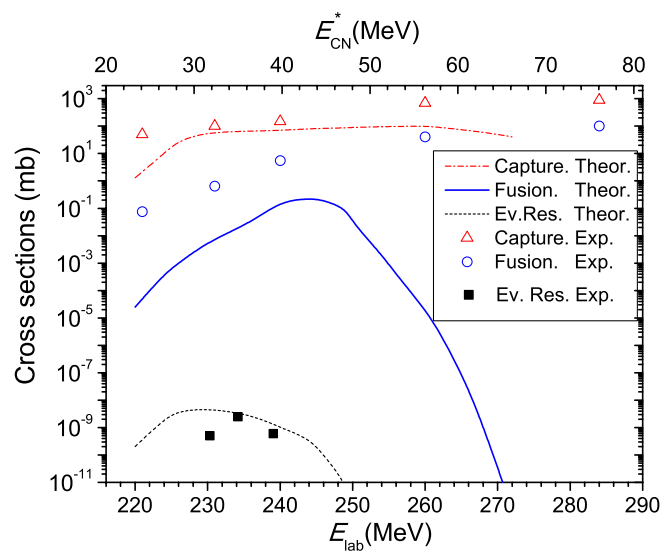

Figure 9. Theoretical capture (dash-dotted curve), fusion (solid curve), and evaporation residue (dashed) excitation functions for the ${ }^{48} \mathrm{Ca}+{ }^{238} \mathrm{U}$ reaction. The experimental cross sections of the evaporation residue are presented by solid squares [18].

the survival probability of the heated $\mathrm{CN}$. The main effects of the use of ${ }^{48} \mathrm{Ca}$ beam are the large fusion cross section encouraging synthesis of the SHE $Z=112-118$ and the decrease of the excitation energy $E_{\mathrm{CN}}^{*}$ which is large in the mass asymmetric reactions. The intrinsic fusion barrier is small for the ${ }^{48} \mathrm{Ca}+{ }^{238} \mathrm{U}$ reaction since the position of the capture point on the PES landscape is placed high as in the case of the ${ }^{34} \mathrm{~S}+{ }^{244} \mathrm{Pu}$ reaction presented in Fig. 4. The fact of the cross sections of the synthesis of the SHE $Z \geq 112$ in the hot reaction become larger than the ones in the cold fusion (see Fig. 1) is the result of the dramatic hindrance to the formation of $\mathrm{CN}$ in the cold fusion ${ }^{70} \mathrm{Zn}+{ }^{208} \mathrm{~Pb}$ (dashed curve in Fig. 8) reaction leading to the $\mathrm{CN}$ with $Z=112$. The large value of $B_{\text {fus }}^{*}$ is a ramatic hindrance to complete fusion. The hot fusion reaction is more favourable in comparison with the cold fusion reaction is explained by the effect of the entrance channel: large fusion cross section in the ${ }^{48} \mathrm{Ca}+{ }^{238} \mathrm{U}$ reaction (see Fig. 9). Therefore, the hot fusion (mass asymmetric) reactions are out of competition in the synthesis of new SHE $Z \geq 112$ since the $B_{\text {fus }}^{*}$ values are relatively small and the condition of the $\mathrm{CN}$ formation is favourable. The dependence of the fission barrier [16] on the neutron numbers in the isotopes of the SHE Cn presented in Fig. 6 shows that the fission barrier of the $\mathrm{CN}{ }^{286} \mathrm{Cn}(N=174)$ formed in the ${ }^{48} \mathrm{Ca}+{ }^{238} \mathrm{U}$ reaction is a little larger $\left(B_{f}=5.01 \mathrm{MeV}\right)$ than the one $\left(B_{f}=4.29 \mathrm{MeV}\right)$ of the $\mathrm{CN}{ }^{278} \mathrm{Cn}$ $(N=166)$ formed in the ${ }^{70} \mathrm{Zn}+{ }^{208} \mathrm{~Pb}$ reaction. The large fusion cross sections in the entrance channel and large fission barriers $B_{f}=6.34$ and $6.07 \mathrm{MeV}$ [16] for the $\mathrm{CN}$ formed in the ${ }^{48} \mathrm{Ca}+{ }^{244} \mathrm{Pu}$ and ${ }^{248} \mathrm{Cm}$ reactions are favourable in the synthesis of $\mathrm{Fl}$ and $\mathrm{Lv}$.

A.K. Nasirov is grateful to the Nobel Foundation for supporting his participation in the Nobel Symposium NS160.

\section{References}

[1] S. Hofmann, Rev. Mod. Phys. 72, 733 (2000).

[2] K. Morita et al., J. Phys. Soc. Jpn. 81, 103201 (2012).

[3] Yu.Ts. Oganessian et al., Phys. Rev. C 69, 054607 (2004).

[4] Yu.Ts. Oganessian et al., Phys. Rev. C 74, 044602 (2006). 
[5] A. Nasirov et al., Nucl. Phys. A759, 342 (2005).

[6] K. Kim et al. Phys. Rev. C 91, 064608 (2015).

[7] G. Fazio et al., J. Phys. Soc. Jpn. 72, 2509 (2003).

[8] A.K. Nasirov et al., Phys. Rev. C 84, 044612 (2011).

[9] A.K. Nasirov et al., Eur. Phys. J. A34, 325 (2007).

[10] A. D’Arrigo et al., J. Phys. G 20, 365 (1994).

[11] R.N. Sagaidak et al., J. Phys. G 24, 611 (1998).

[12] A.K. Nasirov et al., J. Phys.: Conf. Series 205, 012018 (2010).

[13] C. Simenel et al., Phys. Lett. B710, 607 (2012).

[14] P. Möller, J.R. Nix, W.D. Myers, W.J. Swiatecki, At. Data Nucl. Data Tables 59, 185 (1995).

[15] G. Audi and A.H. Wapstra, Nucl. Phys. A595, 509 (1995).

[16] M. Kowal et al. Phys. Rev. C 82, 014303 (2010).

[17] G. Giardina et al. Eur. Phys. J. A8, 2056 (2000).

[18] Yu.Ts. Oganessian et al. Phys. Rev. C 70, 064609 (2004). 